\title{
The alternative lengthening of telomere phenotype is significantly associated with loss of ATRX expression in high-grade pediatric and adult astrocytomas: a multi-institutional study of 214 astrocytomas
}

Malak Abedalthagafi1,2, Joanna J Phillips ${ }^{1,3}$, Grace E Kim² ${ }^{2}$ Sabine Mueller ${ }^{4}$, Daphne A Haas-Kogen ${ }^{5}$, Roxanne E Marshall ${ }^{1}$, Sidney E Croul ${ }^{6}$, Mariarita R Santi ${ }^{7}$, Jing Cheng ${ }^{8}$, Shengmei Zhou ${ }^{9}$, Lisa M Sullivan, Maria Martinez-Lage ${ }^{7}$, Alexander R Judkins ${ }^{9}$ and Arie Perry ${ }^{1,3}$

${ }^{1}$ Division of Neuropathology, Department of Pathology, University of California, San Francisco, San Francisco, CA, USA; ${ }^{2}$ Division of Surgical Pathology, Department of Pathology, University of California, San Francisco, San Francisco, CA, USA; ${ }^{3}$ Department of Neurological Surgery, University of California, San Francisco, San Francisco, CA, USA; ${ }^{4}$ Department of Pediatrics, University of California, San Francisco, San Francisco, CA, USA; ${ }^{5}$ Department of Radiation Oncology, University of California, San Francisco, San Francisco, CA, USA; ${ }^{6}$ Department of Lab Medicine and Pathobiology, University of Toronto, Toronto, Canada; ${ }^{7}$ Department of Pathology and Laboratory Medicine, The Children's Hospital of Philadelphia, Philadelphia, PA, USA; ${ }^{8}$ Division of Epidemiology and Biostatistics, The Clinical and Translational Science Institute (CTSI), University of California, San Francisco (UCSF), San Francisco, CA, USA and ${ }^{9}$ Department of Pathology and Laboratory Medicine, Children's Hospital Los Angeles, Los Angeles, CA, USA

Loss-of-function of alpha thalassemia/mental retardation syndrome X-linked (ATRX) protein leads to a phenotype called alternative lengthening of telomeres (ALT) in some tumors. High-grade astrocytomas comprise a heterogeneous group of central nervous system tumors. We examined a large cohort of adult (91) and pediatric $(n=88)$ high-grade astrocytomas as well as lower grade forms $(n=35)$ for immunohistochemical loss of ATRX protein expression and the presence of ALT using telomere-specific fluorescence in situ hybridization, with further correlation to other known genetic alterations. We found that in pediatric high-grade astrocytomas, $29.6 \%$ of tumors were positive for ALT and $\mathbf{2 4 . 5} \%$ were immunonegative for the ATRX protein, these two alterations being highly associated with one another $(P<0.0001)$. In adult high-grade astrocytomas, $26.4 \%$ of tumors were similarly positive for ALT, including $80 \%$ of ATRX protein immunonegative cases $(P<0.0001)$. Similar frequencies were found in 11 adult low-grade astrocytomas, whereas all 24 pilocytic astrocytomas were negative for ALT. We did not find any significant correlations between isocitrate dehydrogenase status and either ALT positivity or ATRX protein expression in our adult high-grade astrocytomas. In both cohorts, however, the ALT positive high-grade astrocytomas showed more frequent amplification of the platelet-derived growth factor receptor alpha gene (PDGFRA; $45 \%$ and 50\%, respectively) than the ALT negative counterparts (18\% and $26 \% ; P=0.03$ for each). In summary, our data show that the ALT and ATRX protein alterations are common in both pediatric and adult highgrade astrocytomas, often with associated PDGFRA gene amplification.

Modern Pathology (2013) 26, 1425-1432; doi:10.1038/modpathol.2013.90; published online 14 June 2013

Keywords: alternative lengthening of telomeres; ATRX; astrocytoma; FISH; glioblastoma; IDH1; PDGFRA

Correspondence: Dr A Perry, MD, Department of Pathology, University of California San Francisco, 505 Parnassus Avenue Room M551, Box 0102, San Francisco, CA 94143 0102, USA. E-mail: Arie.Perry@ucsf.edu

Received 20 February 2013; revised 21 April 2013; accepted 21 April 2013; published online 14 June 2013
Approximately 22000 new cases of brain and central nervous system tumors are diagnosed each year in the United States, and 3000 of those are in children. Pediatric gliomas comprise a clinically, histologically and molecularly heterogeneous group 
of central nervous system tumors. ${ }^{1}$ In addition, gene expression signatures indicate differences between adult and pediatric cases, ${ }^{1-3}$ although they are histologically indistinguishable and uniformly classified by the current WHO classification for central nervous system tumors. ${ }^{4}$

Alpha thalassemia/mental retardation syndrome X-linked (ATRX) protein belongs to the SWI2/ sucrose non-fermenting 2 family of chromatin remodeling proteins. ATRX and death-domain associated protein (DAXX) form a complex that interacts with histone protein H3.3 to assemble chromatin and telomeres. ${ }^{5,6}$ Mutations that inactivate these genes are common in human pancreatic neuroendocrine tumors and central nervous system tumors. ${ }^{3,7-9}$ Loss of ATRX or DAXX function impairs the heterochromatic state of the telomeres, perhaps because of reduced incorporation of chromatin onto H3.3 histones. ${ }^{8}$ This leads to telomere destabilization, which results in a telomerase-independent telomeremaintenance mechanism called alternative lengthening of telomeres (ALT). ALT can lead to immortalization of cells and carcinogenesis ${ }^{10,11}$ and is not seen in normal cells. ${ }^{12}$ Although the majority of authors have found close relationships between ATRX loss and ALT in diffuse gliomas, ${ }^{7,8,13}$ at least one study found no statistically significant association. ${ }^{14}$

A majority of diffuse low-grade and anaplastic adult astrocytomas, as well as secondary forms of glioblastoma, harbor mutations in the genes encoding isocitrate dehydrogenase $I D H 1$ and $I D H 2 .{ }^{14} I D H$ mutations and $A T R X$ gene alterations often appear together in these astrocytomas. ${ }^{14}$ In addition, highgrade astrocytomas that lack ATRX expression and are ALT-positive often have mutations in the $I D H$ genes. ${ }^{13}$ However, in contrast to adult central nervous system tumors, IDH mutations are exceptionally rare in pediatric high-grade astrocytomas. Amplification of the platelet-derived growth factor receptor alpha (PDGFRA) gene occurs in significant subsets of pediatric and adult high-grade astrocytomas. ${ }^{2,15-20}$ As ALT and ATRX mutations are also common in high-grade astrocytomas, particularly in pediatric and young adult populations, we wanted to investigate the association of PDGFRA amplification with alterations in ALT/ATRX in adult and pediatric gliomas. To date, the correlation between ALT and ATRX remains somewhat uncertain, and potential associations between PDGFRA and ALT/ATRX status have not been investigated. Our findings suggest a significant association between ALT positivity and loss of ATRX in both adult and pediatric high-grade astrocytomas and a correlation between ALT and PDGFRA status. It was also prevalent in adult low-grade astrocytomas, as recently confirmed by another group. ${ }^{21} \mathrm{We}$ also found a correlation between ALT and PDGFRA status.

\section{Materials and methods}

\section{Cohort}

Formalin-fixed, paraffin-embedded tumor tissues from a total of 88 pediatric high-grade astrocytomas, 91 adult high-grade astrocytomas, 11 pediatric lowgrade astrocytomas (pilocytic astrocytomas) and 24 adult low-grade astrocytomas were obtained from University of California San Francisco Brain Tumor Research Center Tissue Bank, Children's Hospital, Los Angeles; Department of Lab Medicine and Pathobiology and Department of Surgery, University of Toronto; Department of Pathology and Laboratory Medicine, The Children's Hospital of Philadelphia, Philadelphia, PA, USA; and Department of Pathology and Laboratory Medicine, University of Pennsylvania. An experienced neuropathologist at each institution performed a diagnostic review on their cases using standard WHO criteria. These included both whole tissue and tissue microarray. The tissue microarrays were previously generated and included six of adult high-grade astrocytomas (one from University of Toronto and five from University of California San Francisco) with average core size of $2 \mathrm{~mm}$ and each tissue microarray contained an average of 20-40 cores (including multiple cores from a single tumor), one tissue microarray of adult low-grade astrocytomas consisting of 24 cases with average core size of $2 \mathrm{~mm}$ (University of California San Francisco) and one tissue microarray of pediatric pilocytic astrocytomas with 11 cases with average core size of $2 \mathrm{~mm}$ (University of California San Francisco). All cores with sufficient tumor tissue available were scored and the results of duplicate cores were averaged. Clinical and molecular characteristics of tumors were obtained from the respective institutions if available and included survival from time of initial surgery, age at initial diagnosis, sex and IDH1 mutant protein status (IDH1R132H), which was assessed by IDH1(R132H) immunohistochemistry (H09, Dianova GmbH, Hamburg, Germany).

\section{Immunohistochemical Analysis of Control Pancreatic Neuroendocrine and Central Nervous System Tumors Samples}

ATRX immunolabeling was performed on formalinfixed, paraffin-embedded sections as described previously. ${ }^{7}$ Briefly, heat-induced antigen retrieval was performed in a steamer using CC1 buffer (catalog\# H-3300, Vector Laboratories) for $30 \mathrm{~min}$. Endogenous peroxidase was blocked (catalog\# S2003, Dako) and serial sections were incubated for $1 \mathrm{~h}$ at room temperature in anti-ATRX primary antibody (1:300; catalog\# HPA001906, SigmaAldrich, lot R00473). Following washing, sections were incubated for $30 \mathrm{~min}$ with horseradish peroxidase-labeled secondary antibody (catalog\# PV6119, Leica Microsystems) followed by 
detection with $3,3^{\prime}$ diaminobenzidine (SigmaAldrich), counterstaining with Harris hematoxylin, rehydration and mounting. Only nuclear labeling was evaluated. One pathologist assessed the immunolabeled pancreatic neuroendocrine tumors specimens (MA).

The study cohort consisted of 88 pediatric highgrade astrocytomas, 24 pediatric pilocytic astrocytomas, 91 adult high-grade astrocytomas and 11 adult low-grade WHO grade II astrocytomas. Highgrade astrocytomas included those diagnosed as WHO grade IV glioblastomas and WHO grade III anaplastic astrocytomas. Owing to the limited availability of tissue, ALT was first performed on all available cases (88 pediatric high-grade astrocytomas, 91 adult high-grade astrocytomas, 11 adult low-grade astrocytomas and 24 pilocytic astrocytoma) and then, ATRX was performed on as many remaining cases as possible (77/88 pediatric high-grade astrocytomas and 74/91 of adult highgrade astrocytomas, 11 adult low-grade astrocytomas, 24 pediatric pilocytic; Tables 1 and 2). Internal positive controls for ATRX included endothelial cells within intratumoral vessels and non-neoplastic neurons. Both cell types demonstrated strong nuclear immunolabeling.

\section{Telomere-Specific and PDGFRA FISH and Microscopy}

The commercially available Telomere FISH Kit/Cy3 (DAKO, catalog code K5326) was used according to the manufacturer's instructions. Briefly, after

Table 1 ALT FISH—by age group and HG or LG

\begin{tabular}{lrrrr} 
& \multicolumn{2}{c}{ ALT FISH } \\
\cline { 3 - 4 } & & Negative & Positive & P-value \\
\cline { 3 - 4 } & $\mathrm{n}$ & & & \\
Adult & & & & \\
HG & 91 & $67(73.6 \%)$ & $24(26.4 \%)$ & 1.0000 \\
LG & 11 & $8(72.7 \%)$ & $3(27.3 \%)$ & \\
Children & & & & \\
HG & 88 & $62(70.5 \%)$ & $26(29.6 \%)$ & $8.937 \mathrm{E}-04$ \\
LG & 24 & $24(100 \%)$ & $0(0 \%)$ & \\
\hline
\end{tabular}

Table 2 ATRX IHC-by age and HG or LG

\begin{tabular}{|c|c|c|c|c|}
\hline & \multirow[b]{2}{*}{$\mathrm{n}$} & \multicolumn{2}{|c|}{ ATRX IHC } & \multirow[b]{2}{*}{ P-value } \\
\hline & & Lost & Present & \\
\hline \multicolumn{5}{|l|}{ Adult } \\
\hline HG & 74 & $15(20.3 \%)$ & $59(79.7 \%)$ & \multirow[t]{2}{*}{0.681} \\
\hline LG & 11 & $1(9.1 \%)$ & $10(90.9 \%)$ & \\
\hline \multicolumn{5}{|c|}{ Children } \\
\hline HG & 77 & $19(24.5 \%)$ & $58(75.3 \%)$ & \multirow[t]{2}{*}{0.010} \\
\hline PA & 21 & $0(0 \%)$ & $21(100 \%)$ & \\
\hline
\end{tabular}

pre-treatment with formaldehyde and a solution containing proteinase $\mathrm{K}$ for $10 \mathrm{~min}$, the sample DNA was denatured at $80{ }^{\circ} \mathrm{C}$ for 4 min under a coverslip in the presence of the Cy3-conjugated probe. Hybridization ( $1 \mathrm{~h}$ at room temperature) was followed by two washes with solutions provided in the kit. Afterward, sections were mounted in antifade reagent containing $4^{\prime}$, 6-diamidino-2-phenylindole as counterstain.

PDGFRA FISH was performed and scored as previously described. ${ }^{20}$ Briefly, unstained slides were deparaffinized by heating them at $70^{\circ} \mathrm{C}$ for $15 \mathrm{~min}$, and then placed in two 10-min washes with Citrisolv, three 3-min washes in isopropanol, and a 20-min wash in 0.2 N HCL. The slides were rinsed in running water for $10 \mathrm{~min}$. DNA target retrieval was achieved by immersing the slides in citrate buffer for $30 \mathrm{~min}$ at $80{ }^{\circ} \mathrm{C}$. Slides were rinsed in running water for $10 \mathrm{~min}$. For tissue digestion, the slides were placed in pepsin (P-7012, Sigma-Aldrich; $2.5 \mathrm{mg} / \mathrm{ml}$ in water) for $25 \mathrm{~min}$ at $37^{\circ} \mathrm{C}$ followed by a $10-\mathrm{min}$ rinse under running water. The slides were then placed in $2 \times \mathrm{SSC}$ for $5 \mathrm{~min}$. A rhodamine-labeled PDGFRA probe was diluted 1:10 in DenHyb (Insitus Biotechnologies, Albuquerque, NM, USA), and the CEP 4 reference probe (Abbott Molecular) was diluted 1:25 in DenHyb. The combined probes were applied to the tissue and co-denatured with the target DNA at $88^{\circ} \mathrm{C}$ for $12 \mathrm{~min}$. The slides were then transferred to a humidified slide moat and incubated overnight at $37^{\circ} \mathrm{C}$. The next day, slides were placed in 50\% formamide/50\% $2 \times$ SSC for $5 \mathrm{~min}$ to remove the coverslips followed by two 10 -min washes in $2 \times$ SSC. Depending on the surface area of the tissue, 10-20 $\mu \mathrm{l}$ of $4^{\prime}, 6$-diamidino-2phenylindole in Fluorguard (Insitus) was placed on the section followed by a coverslip. Signals were observed with an Olympus BX 41 fluorescence microscope.

The ALT FISH and immunolabeled slides were independently assessed and scored by three authors (MA, RM and AP). Large, ultra-bright telomere repeat DNA aggregates defined ALT-positive cell populations as opposed to the small delicate signals derived from normal individual telomeres. ALTpositive cases were identified by large, very bright telomere FISH signals in $\geq 1 \%$ of the tumor cells. A tumor was considered ALT negative if at least 500 consecutive cells lacked large ultrabright telomeric signals. In all cases, areas exhibiting necrosis were excluded from analysis.

\section{Statistical Analysis}

Correlations between categorical variables were analyzed with $\chi^{2}$ or Fisher's exact tests. Overall survival was examined by Cox or Kaplan-Meier tests. $P$-values $<0.05$ were considered statistically significant. All statistical analyses were performed by one author (JC). 


\section{Study Approval}

Clinical data were obtained from University of California San Francisco, University of Toronto, Children's Hospital Los Angeles, and Children's Hospital of Pennsylvania medical records in accordance with Institutional Review Board-approved protocols from each of these institutions.

\section{Results}

Validation of Pancreatic Neuroendocrine Tumors Immunocytochemistry and Telomere-Specific FISH

We first examined 30 sporadic non-functional pancreatic neuroendocrine tumors for technical validation and to provide appropriate positive and negative controls for subsequent experiments. In all, 10 of the 30 pancreatic neuroendocrine tumors $(33.3 \%)$ were ALT positive and had ATRX or DAXX loss according to immunohistochemistry (Figure 1).

\section{Association of ALT and ATRX Loss in Pediatric High-Grade Astrocytomas}

A total of 26 of 88 pediatric high-grade astrocytomas $(29.6 \%)$ were ALT positive (Figure 2a, Table 1). In contrast, none of the 24 pilocytic astrocytomas were ALT positive. We examined 77 of the 88 tumors for ATRX staining. Although 24.5\% (19/77) of pediatric high-grade astrocytomas showed loss of ATRX staining (Table 2, Figure 2b), expression was retained in all 24 pilocytic astrocytomas. Among 19 high-grade astrocytomas in which ATRX was lost, $16(84.2 \%)$ were ALT positive (Table 3$)$. Thus, loss of ATRX staining was significantly associated with ALT in pediatric high-grade astrocytomas $(P<0.0001)$. Further stratification of high-grade tumors into WHO grade III vs IV showed significant associations between ALT positivity and ATRX loss within pediatric anaplastic astrocytoma and glioblastomas subset (Table 4).

\section{Association of ALT and ATRX Loss in Adult High-Grade Astrocytomas}

Telomere-specific FISH analysis in adult high-grade astrocytomas identified ALT positivity in 24 of 91 cases (26.4\%; Table 1). Similar results were observed in adult low-grade diffuse astrocytomas in which 3 of $11(27.3 \%)$ were ALT positive. We examined 74 of the 91 tumors for ATRX. ATRX staining was absent in 15 of 74 (20.3\%) high-grade astrocytomas and 1 of 11 (9.1\%) low-grade WHO grade II astrocytomas (Table 2). Among 15 highgrade astrocytomas in which ATRX was lost, 12 $(80 \%)$ were ALT positive (Table $3 ; P<0.0001)$. ATRX loss was not associated with ALT in low-grade adult astrocytomas, which may reflect the small sample size and the rarity of ATRX immunonegativity.
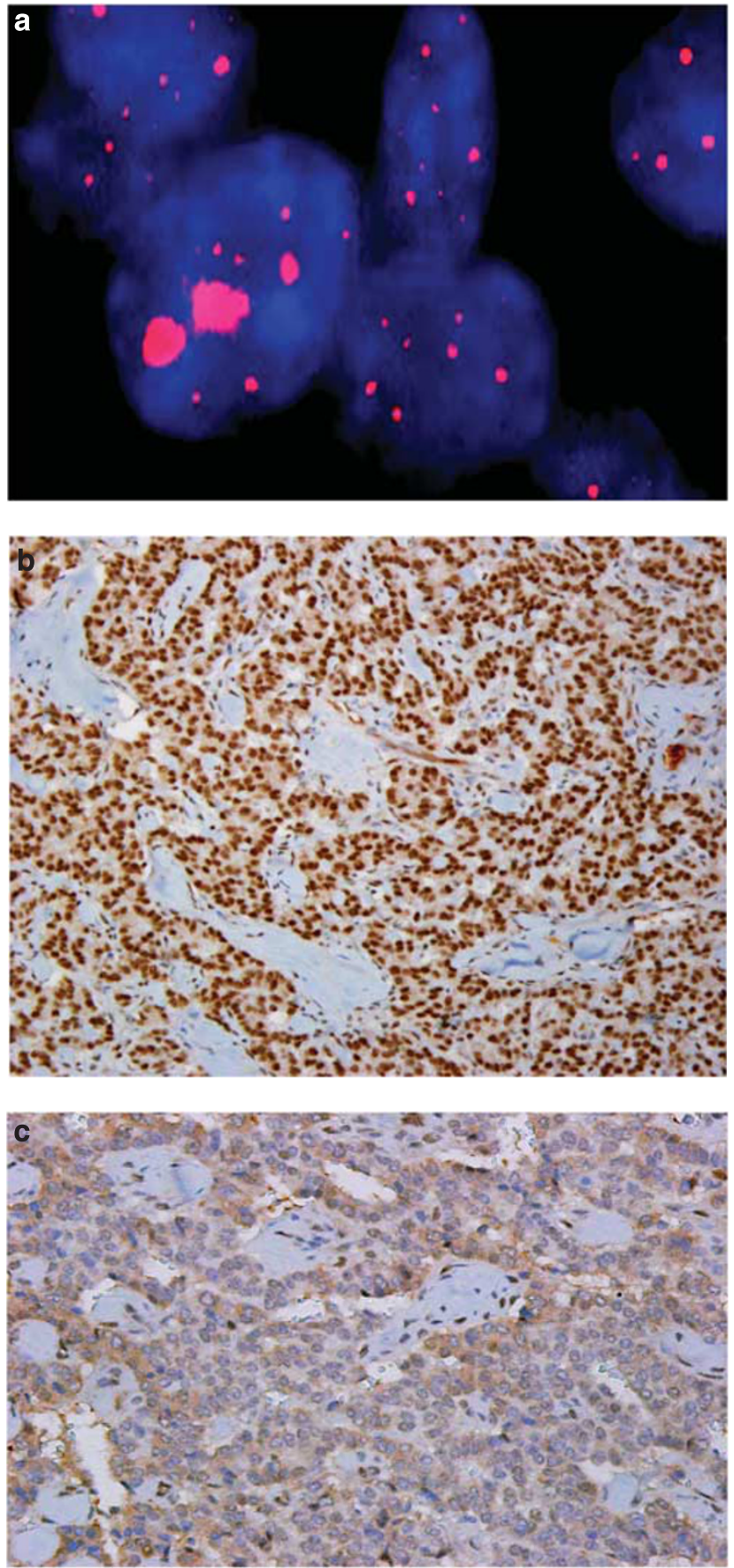

Figure 1 Representative ALT-positive pancreatic neuroendocrine tumors control. (a) Telomere-specific FISH of this tumor shows the ALT-positive phenotype (large, ultrabright telomere FISH signals (red)). (b) Immunolabeling of pancreatic neuroendocrine tumors shows intact nuclear ATRX protein in neoplastic cells. (c) Immunolabeling of the same pancreatic neuroendocrine tumors in panel a shows loss of nuclear ATRX protein in neoplastic cells.

The associations between ALT positivity and ATRX loss in high-grade astrocytomas remained statistically significant with sub-stratification into adult anaplastic astrocytoma and glioblastoma subsets $(P<0.0001$; Table 4). 

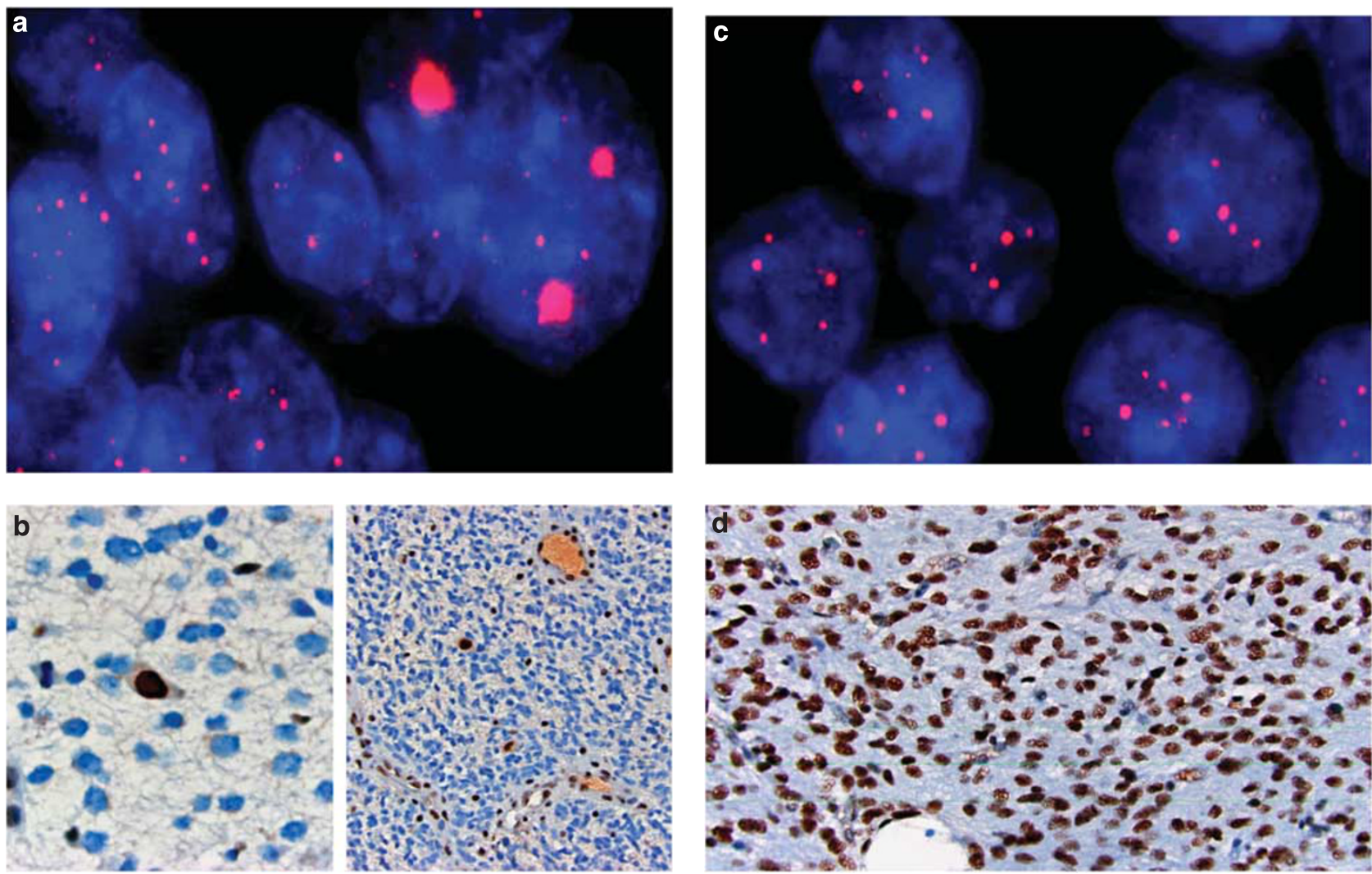

Figure 2 Representative ALT-positive, ATRX-immunonegative glioblastomas. (a) Example of an ALT-positive glioblastoma (large, ultrabright telomere FISH signals (red)). (b) Immunolabeling of the same glioblastoma in panel a shows loss of nuclear ATRX protein in neoplastic cells. (c) Example of an ALT-negative glioblastoma. (d) Immunolabeling of the same glioblastoma in panel c shows intact nuclear ATRX protein in neoplastic cells.

\section{ALT/ATRX and IDH1 Status in Adult High-Grade Astrocytomas}

A total of 72 adult high-grade astrocytomas were examined for IDH1 (R132H) mutation status. In all, 26 of 72 adult high-grade astrocytomas (26.1\%) stained positive for the IDH1 mutant, whereas $72.7 \%(8 / 11)$ of adult low-grade WHO grade II astrocytomas were IDH1 mutant (Table 5). Our data suggested a trend between IDH1 R132H immunopositivity and either ALT positivity or ATRX loss, although these correlations did not reach statistical significance (Table 5; $P=0.116$ and $P=0.115$, respectively).

\section{Associations Between PDGFRA Amplification and ALT/ATRX Status in Pediatric and Adult High-Grade Astrocytomas}

Table 6 summarizes the relationship between ALT and PDGFRA amplification in both adult and pediatric high-grade astrocytomas. In adult highgrade astrocytomas, $50 \%$ (9/18) of ALT-positive cases harbored PDGFRA amplification, which was significantly higher than the 26\% (14/54) amplification rate seen in ALT-negative tumors $(P=0.031)$. A similar statistically significant association was found in pediatric high-grade astrocytomas, in which $45 \%(9 / 20)$ of amplified tumors were ALTpositive and $18 \%(7 / 40)$ of ALT-negative tumors had PDGFRA amplification $(P=0.032)$. ATRX loss was also more common in PDGFRA-amplified adult and pediatric high-grade astrocytomas. However, these associations did not reach statistical significance, yielding $P$-values of 0.181 and 0.195 , respectively, (Table 7).

\section{Survival Analysis}

Survival data in reference to PDGFRA status have been reported in part previously. ${ }^{20}$ Follow-up data until time of death was obtained in 83 (91\%) adult and $55(66 \%)$ pediatric high-grade astrocytoma patients, with median follow-up times of 474 and 419 days, respectively. Median age in years in adult and pediatric high-grade astrocytoma patients with ALT positive/ATRX loss phenotype was 40.7 and 10.3, respectively. Median age in years for highgrade astrocytomas of ALT negative/ATRX intact phenotype was 46.7 in adult and 6.3 in pediatric. No statistically significant associations were found between the ALT or ATRX status (alone or combined) and overall survival in either age group. 
Table 3 ALT FISH and ATRX IHC—by age group and HG or LG

\begin{tabular}{|c|c|c|c|c|}
\hline \multirow[b]{2}{*}{ ALT FISH } & \multicolumn{2}{|c|}{ ATRX IHC } & \multirow[b]{2}{*}{ Total } & \multirow[b]{2}{*}{$\mathrm{P}$-value } \\
\hline & Absent & Present & & \\
\hline \multicolumn{5}{|l|}{ Adult $H G$} \\
\hline Negative & 3 & 54 & 57 & $<0.0001$ \\
\hline Positive & 12 & 5 & 17 & \\
\hline Total & 15 & 59 & 74 & \\
\hline \multicolumn{5}{|l|}{ Adult $L G$} \\
\hline Negative & 0 & 8 & 8 & 0.273 \\
\hline Positive & 1 & 2 & 3 & \\
\hline Total & 1 & 10 & 11 & \\
\hline \multicolumn{5}{|l|}{ Pediatric $H G$} \\
\hline Negative & 3 & 49 & 52 & $<0.0001$ \\
\hline Positive & 16 & 9 & 25 & \\
\hline Total & 19 & 58 & 77 & \\
\hline \multicolumn{5}{|l|}{ Pediatric $L G$} \\
\hline Negative & 0 & 24 & 24 & \\
\hline Positive & 0 & 0 & 0 & \\
\hline Total & 0 & 24 & 24 & \\
\hline
\end{tabular}

Similarly, combinations of ALT status with IDH1 or PDGFRA data were not statistically associated with overall survival times in our cohort.

\section{Discussion}

Recent studies have shown conflicting data regarding the association between ATRX loss and ALT status in glioblastomas. ${ }^{13,14}$ Our data suggest that the ALT phenotype is highly associated with ATRX loss in high-grade astrocytomas of both age groups $(P<0.0001)$, supporting the model in which loss of ATRX function impairs the heterochromatic state of telomeres, perhaps because of reduced levels of histone H3.3 incorporation; this subsequently leads to telomere destabilization and increased homologous recombination, thus facilitating the development of ALT. ${ }^{8,22}$ Abnormally long telomeres are known to be associated with cell immortality and cancer. ${ }^{10,11,23}$ As such, telomeretargeted therapies should be studied further for their potential benefits in patients with ALT-positive tumors. Although telomerase inhibition may result in adverse toxicity to bone marrow or other progenitor cell populations, ${ }^{24}$ this strategy may nonetheless benefit many cancer patients. Telomerase knockdown strategies or viruses that target telomerase could also limit the survival of cancer cells that show telomere dysfunction, including those with ALT.

A recent study proposed six biological classification subgroups of glioblastoma based on global DNA methylation patterns. ${ }^{3}$ Most patients whose tumors were characterized by the protein complex of G34 or K27 mutant histone H3.3 and ATRX clustered in the
Table 4 By age group and A2, AA, GBM or pilocytic astrocytoma

ATRX IHC

ALT FISH Absent Present Total P-value Breslow-Day CMH

\begin{tabular}{|c|c|c|c|c|c|c|}
\hline \multicolumn{7}{|l|}{ Adult A2 } \\
\hline Negative & 0 & 8 & 8 & 0.300 & 0.738 & $<0.0001$ \\
\hline Positive & 1 & 2 & 3 & & & \\
\hline Total & 1 & 10 & 11 & & & \\
\hline \multicolumn{7}{|l|}{ Adult $A A$} \\
\hline Negative & 0 & 6 & 6 & 0.024 & & \\
\hline Positive & 3 & 1 & 4 & & & \\
\hline Total & 3 & 7 & 10 & & & \\
\hline \multicolumn{7}{|l|}{ Adult GBM } \\
\hline Negative & 3 & 48 & 51 & $<0.0001$ & & \\
\hline Positive & 9 & 4 & 13 & & & \\
\hline Total & 12 & 52 & 64 & & & \\
\hline \multicolumn{7}{|c|}{ Pediatric AA } \\
\hline Negative & 0 & 26 & 26 & $<0.0001$ & 0.021 & $<0.0001$ \\
\hline Positive & 9 & 2 & 11 & & & \\
\hline Total & 9 & 28 & 37 & & & \\
\hline \multicolumn{7}{|c|}{ Pediatric GBM } \\
\hline Negative & 3 & 23 & 26 & 0.018 & & \\
\hline Positive & 7 & 7 & 14 & & & \\
\hline Total & 10 & 30 & 40 & & & \\
\hline \multicolumn{7}{|c|}{ Pediatric pilocytic astrocytoma } \\
\hline Negative & 0 & 24 & 24 & & & \\
\hline Positive & 0 & 0 & 0 & & & \\
\hline Total & 0 & 0 & 24 & & & \\
\hline
\end{tabular}

adolescent and young adult populations. Although we did not test the H3.3 mutation status in our cohort, we did not find ATRX losses to be restricted to young adults (the range of age between 20 and 79 with a median of 40.7 years) or adolescents (the range age for the pediatric group was between 5 and 17 with a median of 10.3 years).

In contrast to the study by Nguyen et al, ${ }^{13}$ we did not find a significant improvement in survival for ALT-positive high-grade astrocytoma patients in our cohort.

Amplification of the PDGFRA gene is also present in a significant subset of pediatric and adult highgrade astrocytomas. ${ }^{2,15-20}$ Of interest, our study identified a significant association between PDGFRA amplification and ALT positivity in both adult and pediatric high-grade astrocytomas $(P=0.03)$. The biological explanation for this association is not clear, but suggests that perhaps inhibition of the PDGFRA signaling pathway may be beneficial in this subgroup of patients. Further studies exploring this association are therefore warranted.

In summary, the ALT phenotype and ATRX losses identify significant biological subsets of high-grade astrocytoma in both adult and pediatric patients. Our results show that telomere-specific FISH and ATRX staining are reliable detection assays in 
Table 5 ALT and IDH status

\begin{tabular}{|c|c|c|c|c|}
\hline \multirow[b]{2}{*}{ ALT FISH } & \multicolumn{2}{|c|}{ IDH status } & \multirow[b]{2}{*}{ Total } & \multirow[b]{2}{*}{$\mathrm{P}$-value } \\
\hline & $I D H+$ & $I D H-$ & & \\
\hline \multicolumn{5}{|l|}{ Adult $H G$} \\
\hline Negative & 17 & 41 & 58 & 0.116 \\
\hline Positive & 9 & 5 & 14 & \\
\hline Total & 26 & 46 & 72 & \\
\hline \multicolumn{5}{|l|}{ Adult $L G$} \\
\hline Negative & 6 & 2 & 8 & 1.00 \\
\hline Positive & 2 & 1 & 3 & \\
\hline \multirow[t]{2}{*}{ Total } & 8 & 3 & 11 & \\
\hline & \multicolumn{2}{|c|}{ IDH status } & & \\
\hline ATRX IHC & $I D H+$ & $I D H-$ & Total & $\mathrm{P}$-value \\
\hline \multicolumn{5}{|c|}{ ATRX IHC and IDH status } \\
\hline \multicolumn{5}{|l|}{ Adult $H G$} \\
\hline Lost & 7 & 1 & 8 & 0.115 \\
\hline Present & 19 & 17 & 36 & \\
\hline Total & 26 & 18 & 44 & \\
\hline \multicolumn{5}{|l|}{ Adult $L G$} \\
\hline Lost & 1 & 0 & 1 & 1.00 \\
\hline Present & 7 & 3 & 10 & \\
\hline Total & 8 & 3 & 11 & \\
\hline
\end{tabular}

Table 6 ALT FISH and PDGFR combined-by age group and HG, LG

\begin{tabular}{lrrrr} 
& \multicolumn{2}{c}{ PDGFR } & & \\
\cline { 2 - 3 } ALT FISH & AMP & Non-AMP & Total & P-value \\
\hline Adult HG & & & & \\
$\quad$ Negative & 14 & 40 & 54 & 0.031 \\
Positive & 9 & 9 & 18 & \\
Total & 23 & 49 & 72 & \\
Pediatric $H G$ & & & & \\
$\quad$ Negative & 7 & 33 & 40 & 0.032 \\
$\quad$ Positive & 9 & 11 & 20 & \\
$\quad$ Total & 16 & 44 & 60 & \\
\end{tabular}

formalin-fixed, paraffin-embedded tissue with 80-84\% concordance. There are a number of potential explanations for non-concordant cases. For example, some missense ATRX mutations would not be detected by routine immunohistochemistry. ALT may also occur because of mutations of other genes, such as $D A X X$, although this appears to be rare in gliomas. We also used strict criteria in scoring the ATRX loss, which we defined as being $100 \%$ absent in tumor nuclei. This approach is more likely to be feasible and reproducible, allowing establishment of ATRX loss as a clinical biomarker. Thus, ATRX IHC alone can underestimate mutation status and ALT incidence. In terms of deciding which assay to use in a clinical or routine pathology
Table 7 ATRX IHC and PDGFR combined

\begin{tabular}{lrrrr}
\hline & \multicolumn{2}{c}{ PDGFR } & & \\
\cline { 2 - 3 } ATRX IHC & AMP & Non-AMP & Total & P-value \\
\hline Adult HG & & & & \\
$\quad$ Absent & 6 & 5 & 11 & 0.181 \\
Present & 14 & 30 & 44 & \\
Total & 20 & 35 & 55 & \\
& & & & \\
Pediatric HG & & 10 & 17 & 0.195 \\
$\quad$ Absent & 7 & 33 & 42 & \\
Present & 9 & 43 & 59 & \\
$\quad$ Total & 16 & & & \\
\hline
\end{tabular}

setting, immunohistochemistry is simpler, cheaper and more readily available. Nevertheless, given that ALT is the end product determining aberrant telomere maintenance, the FISH assay may be of greater biological relevance. Finally, our study found potential interactions between ALT/ATRX and other genetic alterations such as PDGFRA amplification, which should be explored further in the development of individualized treatment strategies.

\section{Acknowledgements}

This work was supported in part by the National Institutes of Health, (R01 NS081117 to JP), UCSF Brain Tumor SPORE (CA097257), the James S McDonnell Foundation (JP) and funds from the University of California San Francisco Departments of Pathology and Neurological Surgery. These data were presented in part at the 102nd USCAP Annual Meeting, Baltimore, MD, USA, 4 March 2013.

\section{Disclosure/conflict of interest}

The authors declare no conflict of interest.

\section{References}

1 Biegel JA, Pollack IF. Molecular analysis of pediatric brain tumors. Curr Oncol Rep 2004;6:445-452.

2 Paugh BS, Qu C, Jones C, et al. Integrated molecular genetic profiling of pediatric high-grade gliomas reveals key differences with the adult disease. J Clin Oncol 2010;28:3061-3068.

3 Sturm D, Witt H, Hovestadt V, et al. Hotspot mutations in H3F3A and IDH1 define distinct epigenetic and biological subgroups of glioblastoma. Cancer Cell 2012;22:425-437.

4 Nageswara Rao AA, Packer RJ. Impact of molecular biology studies on the understanding of brain tumors in children. Curr Oncol Rep 2012;14:206-212.

5 Law MJ, Lower KM, Voon HP, et al. ATR-X syndrome protein targets tandem repeats and influences allele- 
specific expression in a size-dependent manner. Cell 2010;143:367-378.

6 Whitehouse I, Owen-Hughes T. ATRX: put me on repeat. Cell 2010;143:335-336.

7 Jiao Y, Shi C, Edil BH, et al. DAXX/ATRX, MEN1, and mTOR pathway genes are frequently altered in pancreatic neuroendocrine tumors. Science 2011;331: 1199-1203.

8 Heaphy CM, de Wilde RF, Jiao $\mathrm{Y}$, et al. Altered telomeres in tumors with ATRX and DAXX mutations. Science 2011a;333:425.

$9 \mathrm{Wu} \mathrm{G}$, Broniscer A, McEachron TA, et al. Somatic histone $\mathrm{H} 3$ alterations in pediatric diffuse intrinsic pontine gliomas and non-brainstem glioblastomas. Nat Gen 2012;44:251-253.

10 Henson JD, Neumann AA, Yeager TR, et al. Alternative lengthening of telomeres in mammalian cells. Oncogene 2002;21:598-610.

11 Durant S. Telomerase-independent paths to immortality in predictable cancer subtypes. J Cancer 2012;3: $67-82$.

12 Heaphy CM, Subhawong AP, Hong SM, et al. Prevalence of the alterative lengthening of telomeres telomere maintenance mechanism in human cancer subtypes. Am J Pathol 2011b;179:1608-1615.

13 Nguyen DN, Heaphy CM, de Wilde RF, et al. Molecular and morphologic correlates of the alternative lengthening of telomeres phenotype in high-grade astrocytomas. Brain Pathol 2013;23:237-243.

14 Liu XY, Gerges N, Korshunov A, et al. Frequent ATRX mutations and loss of expression in adult diffuse astrocytic tumors carrying IDH1/IDH2 and TP53 mutations. Acta Neuropathol 2012;124:615.

15 Szerlip NJ, Pedraza A, Chakravarty D, et al. Intratumoral heterogeneity of receptor tyrosine kinases EGFR and PDGFRA amplification in glioblastoma defines subpopulations with distinct growth factor response. Proc Natl Acad Sci USA 2012;109:3041-3046.

16 Puget S, Philippe C, Bax DA, et al. Mesenchymal transition and PDGFRA amplification/mutation are key distinct oncogenic events in pediatric diffuse intrinsic pontine gliomas. PLoS One 2012;7:e30313.

17 Zarghooni Brennan CW, Wang L, et al. Whole-genome profiling of pediatric diffuse intrinsic pontine gliomas highlights platelet-derived growth factor receptor alpha and poly potential therapeutic targets. J Clin Oncol 2010;28:1337-1344.

18 Ozawa Brennan CW, Wang L, et al. PDGFRA gene rearrangements are frequent genetic events in PDGFRAamplified glioblastomas. Genes Dev 2010;24:2205-2218.

19 Verhaak RG, Hoadley KA, Purdom E, et al. Integrated genomic analysis identifies clinically relevant subtypes of glioblastoma characterized by abnormalities in PDGFRA, IDH1, EGFR, and NF. Cancer Cell 2010;17: 98-110.

20 Phillips JJ, Aranda D, Ellison DW, et al. PDGFRA amplification is common in pediatric and adult highgrade astrocytomas and identifies a poor prognostic group in IDH1 mutant glioblastoma. Brain Pathol 2013 [e-pub ahead of print].

21 Kannan K, Inagaki A, Silber J, et al. Whole-exome sequencing identifies ATRX mutation as a key molecular determinant in lower-grade glioma. Oncotarget 2012;3:1194-1203.

22 Schwartzentruber J, Korshunov A, Liu XY, et al. Driver mutations in histone H3.3 and chromatin remodeling genes in paediatric glioblastoma. Nature 2012;482: 226-231.

23 Blackburn EH. Structure and function of telomeres. Nature 1991;350:569-573.

$24 \mathrm{Xu} \mathrm{L}$, Li S, Stohr BA. The role of telomere biology in cancer. Ann Rev Pathol Mech Dis 2013;8:49-78. 